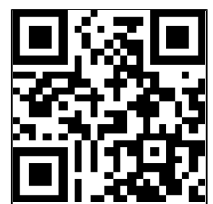

Editor's choic Scan to access more free content

- Additional material is published online only. To view please visit the journal online (http://dx.doi.org/10.1136/ bjsports-2014-094220).

For numbered affiliations see end of article.

\section{Correspondence to} Dr Jonathan T Finnoff, Mayo Clinic, 200 First Street SW, Rochester, MN 55905, USA Finnoff.jonathan@mayo.edu

Accepted 2 October 2014 Published Online First 31 October 2014

\title{
American Medical Society for Sports Medicine recommended sports ultrasound curriculum for sports medicine fellowships
}

\author{
Jonathan T Finnoff, ${ }^{1,2}$ David Berkoff, ${ }^{3}$ Fred Brennan, ${ }^{4}$ John DiFiori, ${ }^{5}$ Mederic M Hall, ${ }^{6}$ \\ Kimberly Harmon, ${ }^{7}$ Mark Lavallee, ${ }^{8,9,10}$ Sean Martin, ${ }^{11}$ Jay Smith, ${ }^{12,13}$ Mark Stovak $^{14}$
}

\section{ABSTRACT}

The American Medical Society for Sports Medicine (AMSSM) developed a musculoskeletal ultrasound curriculum for sports medicine fellowships in 2010. As the use of diagnostic and interventional ultrasound in sports medicine has evolved, it became clear that the curriculum needed to be updated. Furthermore, the name 'musculoskeletal ultrasound' was changed to 'sports ultrasound' (SPORTS US) to reflect the broad range of diagnostic and interventional applications of ultrasound in sports medicine.

This document was created to outline the core competencies of SPORTS US and to provide sports medicine fellowship directors and others interested in SPORTS US education with a guide to create a SPORTS US curriculum. By completing this SPORTS US curriculum, sports medicine fellows and physicians can attain proficiency in the core competencies of SPORTS US required for the practice of sports medicine.

\section{INTRODUCTION}

The following sports ultrasound (SPORTS US) curriculum is a revision of the curriculum developed by the American Medical Society for Sports Medicine (AMSSM) in 2010. ${ }^{1}$ Several changes have been made to the curriculum with the primary aim of providing a pathway by which a sports medicine fellow can obtain sufficient SPORTS US training to become proficient in the core competencies of SPORTS US. The core competencies of SPORTS US are outlined in the learning objectives section of this document.

The term 'SPORTS US' was purposefully chosen rather than 'musculoskeletal ultrasound' (MSK US) since it was recognised by the panel that the evolving field of SPORTS US encompasses non-MSK applications of ultrasound such as the FAST examination (focused assessment with sonography for trauma). While the SPORTS US core competencies in this curriculum are all MSK in nature, they represent the minimum SPORTS US knowledge a sports medicine fellow should acquire during fellowship. However, additional training in more advanced MSK and non-MSK applications of ultrasound can be provided at the fellowship director's discretion.

Completion of this SPORTS US curriculum fulfils the American Institute of Ultrasound in Medicine's (AIUM) requirements to perform a MSK US examination, and the pre-requisites for the American Registry for Diagnostic Medical Sonography's (ARDMS) MSK sonography certification examination.

\section{Overview}

The educational process should include four components.

Didactic instructional sessions

Didactic instruction can occur via a dedicated SPORTS US course or scheduled teaching sessions during the fellowship (see Didactic instructional sessions). Didactics should include discussions of ultrasound physics, image acquisition and optimisation, normal and pathological appearance of tissues, ultrasound artifacts, advantages and limitations of SPORTS US relative to other imaging modalities, and diagnostic and interventional techniques pertaining to major body regions encountered in a sports medicine practice.

Didactic practice sessions

Hands-on didactic practice sessions should be completed under the direct supervision of a qualified mentor. A qualified mentor is defined as an individual who has met the qualifications outlined by the AIUM Training Guidelines for Physicians and Chiropractors Who Evaluate and Interpret Diagnostic Musculoskeletal Ultrasound Examinations (http:// www.aium.org). During these sessions, fellows should apply the knowledge and skills acquired during the didactic instructional sessions in a controlled and supervised environment (see Didactic practice sessions).

\section{Mentored clinical experience}

As knowledge and skills are acquired, the fellow should perform diagnostic scanning and interventional procedures on patients in a clinical setting under the direct supervision of a qualified mentor. As the fellow gains proficiency in the clinical application of diagnostic and interventional SPORTS US techniques, the level of supervision may be modified as allowed by institutional policy governing teaching rules.

\section{Supplementary and continuing education}

The fellow's education should include supplementary educational experiences to reinforce the knowledge and skills gained during the didactic sessions and mentored clinical experience.

A. Required

1. Independent scanning practice sessions

B. Recommended

1. Reading reference texts and journal article

2. Presenting SPORTS US related articles in journal club 
3. Utilising online educational material and educational DVD's

4. Attending SPORTS US conferences and presentations

The integration of recommended supplementary educational experiences may vary from fellowship to fellowship based on available resources and the overall curriculum structure.

This natural stepwise progression of diagnostic and interventional SPORTS US education will ensure the acquisition of sufficient SPORTS US skills to allow independent practice of the core competencies of diagnostic and interventional SPORTS US on completion of fellowship.

\section{Learning objectives for SPORTS US training during fellowship}

A. Identify and discuss the function of basic controls on an ultrasound machine console, including

i. Transducer selection

ii. Presets

iii. Depth

iv. Focal zone/focal region

v. Gain

vi. Time gain compensation/depth gain compensation

vii. Zoom (including read zoom and write zoom)

B. Discuss the basic physics principles of ultrasound, including

i. How an US image is generated

ii. Inter-relationship of machine controls (eg, frequency, resolution and depth)

iii. Doppler imaging (difference between power Doppler and colour Doppler)

C. Demonstrate how to optimise an ultrasound image

i. Superficial structures

ii. Deep structures

D. Describe the normal ultrasonographic appearance of adipose, muscle, tendon, ligament, bone, fascia, vessels, nerve, and cartilage

E. Describe the common pathological ultrasonographic appearance of adipose, muscle, tendon, ligament, bone, fascia, vessels, nerve, joint and cartilage

F. Discuss the benefits and limitations of SPORTS US relative to other imaging modalities

G. Identify and discuss the source and/or implications of basic ultrasound artefacts, including

i. Anisotropy

ii. Reverberation

iii. Refraction

iv. Through transmission

v. Acoustic shadowing

\section{Perform image acquisition of vascular structures including} neovessels using Color and Power Doppler.

H. Perform a SPORTS US examination of the following regions as recommended by the AIUM Practice Guidelines for the Performance of the MSK US Examination (see online supplementary appendix 1)

i. Shoulder

ii. Elbow

iii. Wrist-Hand

iv. Hip

v. Knee

vi. Ankle-Foot

I. Obtain an acceptable set of SPORTS US images of the following regions as recommended by the AIUM Practice Guidelines for the Performance of the MSK US Examination (see online supplementary appendix 1)
i. Shoulder
ii. Elbow
iii. Wrist-Hand
iv. Hip
v. Knee
vi. Ankle-Foot

J. Demonstrate appropriate labelling of SPORTS US images

i. Use of text insertion

ii. Use of arrows and measurement calipers

K. Demonstrate how to capture, store and transfer SPORTS US images

L. Generate an appropriate diagnostic SPORTS US report

M. Perform an appropriate SPORTS US evaluation to identify and appropriately document (eg, capture, label, save and transfer images; generate a report) of the following conditions:

i. Shoulder

1. Supraspinatus full thickness tear

2. Supraspinatus tendinopathy

3. Bicipital tendinopathy

4. Subacromial-subdeltoid bursopathy

5. Acromioclavicular joint osteoarthritis

ii. Elbow

1. Common extensor tendinopathy

2. Dynamic examination of the ulnar nerve at the elbow

3. Common flexor tendinopathy

iii. Wrist-Hand

1. DeQuervain's tenosynovitis

2. Carpal tunnel syndrome

iv. Hip

1. Gluteus medius/minimus tendinopathy

2. Hamstring tendinopathy

v. Knee

1. Patellar tendinopathy

2. Baker's cyst

3. Knee joint effusion

vi. Ankle-Foot

1. Peroneal tendinopathy (including dynamic evaluation for instability)

2. Achilles tendinopathy

3. Plantar fasciopathy

N. Describe the advantages and disadvantages of needle tracking using an in-plane versus out-of-plane approach, and provide clinical examples of when each approach may be beneficial.

O. Image a needle using an in-plane (longitudinal or long axis) and out-of-plane (short axis or transverse) approach using ultrasound guidance in a phantom, turkey breast, cadaveric specimen or other imaging medium, including demonstration of the following transducer manipulations:

i. Translation (sliding/gliding)

ii. Rotation

1. Describe 'cross-cut' artefact when imaging/tracking a needle during an interventional procedure

iii. Heel-toe

iv. Tilting (toggling/wagging)

v. Compression

P. Demonstrate the ability to efficiently relocate a lost needle during an in-plane and out-of-plane needle tracking approach.

Q. Demonstrate the ability to guide a needle into a target region or structure using an in-plane and out-of-plane approach in a phantom, turkey breast, cadaveric specimen or other imaging medium. 
R. Obtain an acceptable set of preprocedure, intraprocedure and postprocedure images of an ultrasound-guided-procedure.

S. Demonstrate appropriate labelling of the ultrasound-guided procedure images.

T. Demonstrate how to store and transfer the ultrasoundguided procedure images.

U. Generate an appropriate ultrasound-guided procedure report.

V. Perform and appropriately document (eg, capture, label, save, and transfer images; generate a report) the following ultrasound-guided procedures:

i. Shoulder

1. Subacromial-subdeltoid bursa injection

2. Intra-articular glenohumeral joint injection

3. Intra-articular acromioclavicular joint injection

4. Bicipital tendon sheath injection

ii. Elbow

1. Intra-articular elbow joint injection

2. Peritendinous or intratendinous injection of the common extensor tendon origin

3. Peritendinous or intratendinous injection of the common flexor tendon origin

iii. Wrist-Hand

1. Carpal tunnel injection

2. First dorsal compartment tendon sheath injection

3. Intra-articular wrist injection

iv. Hip

1. Intra-articular hip injection

2. Greater trochanteric bursa injection

3. Gluteus medius or minimus peritendinous or intratendinous injection

v. Knee

1. Intra-articular knee injection

2. Iliotibial band/bursa (distal) injection

vi. Ankle-Foot

1. Intra-articular tibiotalar joint injection

2. Peroneal tendon sheath injection

3. Periplantar or intraplantar fascia injection

vii. Miscellaneous

1. Aspiration or injection of a cyst

\section{Resources/references}

Books

Bianchi S, Martinoli C. Ultrasound of the musculoskeletal system. New York: Springer, 2007.

Daniels JM, Dexter WW, eds. Basics of musculoskeletal ultrasound. New York: Springer, 2013.

Jacobson JA. Fundamentals of musculoskeletal ultrasound. 2nd edn. Philadelphia: Saunders Elsevier, 2013.

Malanga G, Mautner K, eds. Atlas of ultrasound-guided musculoskeletal injections. New York: McGraw-Hill, 2014.

O'Neill J, ed. Musculoskeletal ultrasound: anatomy and technique. New York: Springer, 2008.

Sconfienza LM, Serafini G, Silvestri E, eds. Ultrasound guided musculoskeletal procedures: the upper limb. New York: Springer, 2012.

Silvestri E, Muda A, Sconfienza LM, eds. Normal ultrasound anatomy of the musculoskeletal system: a practical guide. New York: Springer, 2012.

\section{Additional resources}

American College of Radiology (ACR) Practice Guideline for Communication of Diagnostic Imaging Findings. http://www.acr.org
ACR Practice Guidelines for the Performance of the Musculoskeletal Ultrasound Examination. http://www.acr.org American Institute of Ultrasound in Medicine (AIUM) Practice Guidelines for the Performance of the Musculoskeletal Ultrasound Examination, 2012. http://www.aium.org, or in the J Ultrasound Med 2012;31:1480.

AIUM Practice Guideline for the Performance of the Shoulder Ultrasound Examination. http://www.aium.org

AIUM Practice Guideline for Documentation of the Diagnostic Ultrasound Examination. http://www.aium.org

AIUM Practice Guideline for Performance of the Diagnostic Ultrasound Examination. http://www.aium.org

AIUM Training Guidelines for Physicians and Chiropractors Who Evaluate and Interpret Diagnostic Musculoskeletal Ultrasound Examinations. http://www.aium.org

European Society of Skeletal Radiology (ESSR) Musculoskeletal Ultrasound Scanning Technique Demonstrations. http://www. ESSR.org

Smith J, Finnoff JT. Diagnostic and interventional musculoskeletal ultrasound: part 1. Fundamentals. PMR 2009;1:64-75.

Smith J, Finnoff JT. Diagnostic and interventional musculoskeletal ultrasound: part 2. PMR 2009;1:162-77.

\section{Didactic instructional sessions}

The SPORTS US didactic instructional sessions include six basic units described in this section. Each fellowship should provide appropriate resources for fellows to preview and review the information relevant to each session (see Resource/reference list). Whereas the number of teaching sessions can be modified as desired or necessary, all fellows should receive instruction in all listed topics. It is strongly recommended that teaching sessions for diagnostic scanning (Units 1-5) utilise established scanning protocols to guide learning and ensure compliance with accepted standards (See SPORTS US Scanning Protocol in online supplementary appendix 2). Finally, although the order of Units 2-5 may be modified, fellows should master the diagnostic skills for a specific region before initiating ultrasoundguided procedure training in that region (eg, mastery of the shoulder diagnostic scans should proceed formal training in ultrasound-guided shoulder interventions).

The first unit introduces the fellow to basic SPORTS US physics, image acquisition and optimisation, normal and pathological appearance of tissues, ultrasound artefacts and the advantages and limitations of SPORTS US relative to other imaging modalities. During Units 2-5, a qualified MSK sonographer/sonologist should demonstrate the scanning protocol(s) for one or more body regions, followed by supervised practice. A qualified MSK sonographer is defined as an individual who has met the AIUM Practice Guidelines for the Performance of a MSK US examination. The fellow should only consider these sessions as an introduction to scanning and independent practice between didactic sessions is necessary to facilitate skill acquisition (see Supplementary and continuing education above).

Unit 6 involves at least three individual sessions dedicated to interventional SPORTS US procedures. Initial topics reviewed include pharmacological principles of commonly used medications, patient selection, aseptic technique for ultrasound-guided procedures, procedural risks, and treatment of common adverse events. Thereafter, the fellow should be introduced to methods of ultrasound-guided needle image optimisation, needle relocation and dynamic needle tracking using both in-plane and out-of-plane approaches. Once these skills are mastered, the fellow should be introduced to common upper and lower extremity ultrasound-guided interventional procedures (with a 
focus on the core interventional ultrasound competencies) via discussion, demonstration and supervised practice under the guidance of a qualified practitioner. Multiple mediums may be suitable for practising ultrasound-guided interventional procedures. The ideal mediums are unembalmed cadaveric specimens. However, if unembalmed cadaveric specimens are unavailable, the fellow can practice patient positioning and target acquisition on live models followed by practice of needle visualisation and guidance on turkey breasts, pig feet, pig legs, firm tofu, phantoms and/or other non-cadaveric specimens. The fellow should practice needle visualisation and guidance techniques between mentored didactic sessions to enhance his/her skills (see supplementary and continuing education above). ${ }^{\mathrm{i}}$

Unit 1 Principles of SPORTS US and an Introduction to Scanning Techniques

1. Instruction in 'Knobology' and basic scanning techniques

2. Instruction on basic US physics

3. Demonstration of normal sonographic appearance of adipose, muscle, tendon, ligament, bone, fascia, vessels, nerve, and cartilage

4. Discussion of the common abnormal sonographic appearances of adipose, muscle, tendon, ligament, bone, fascia, vessels, nerve, joint and cartilage

5. Muscle, tendon, ligament and nerve.

6. Demonstration of the use of Color and Power Doppler for imaging vascular and neovascular structures

7. Demonstration of transducer movements to optimise image (translation (sliding), rotation, heel-toe, tilt (toggle) and pressure/compression)

8. Supervised practice

Unit 2 SPORTS US Examination of the Knee-Hip

1. Knee US Scanning Protocol

A. Instruction and supervised practice

B. Resources

i. SPORTS US Scanning Protocol Checklists-Knee

ii. AIUM and ACR Guidelines for Performance of the MSK US Examination

2. Hip and Thigh US Scanning Protocol

A. Instruction and supervised practice

B. Resources

i. SPORTS US Scanning Protocol ChecklistsHip-Thigh

ii. AIUM and ACR Guidelines for Performance of the MSK US Examination

3. Independent scanning

Unit 3 SPORTS US Examination of the Elbow and Wrist-Hand

1. Elbow US Scanning Protocol

A. Instruction and supervised practice

B. Resources

i. SPORTS US Scanning Protocol Checklists-Elbow

ii. AIUM and ACR Guidelines for Performance of the MSK US Examination

2. Wrist-Hand US Scanning Protocol

A. Instruction and supervised practice

B. Resources

${ }^{i}$ Please note that the educational material has been divided into units to facilitate teaching of related concepts and skills. The number of educational sessions required to teach the knowledge and skills contained in a specific unit may vary depending on scheduling and available resources. i. SPORTS US Scanning Protocol ChecklistsWrist-Hand

ii. AIUM Guidelines for Performance of the MSK US Examination

3. Independent scanning

Unit 4 SPORTS US Examination of the Ankle-Foot

1. Ankle-Foot US Scanning Protocol

A. Instruction and supervised practice

B. Resources

i. SPORTS US Scanning Protocol ChecklistsAnkle-Foot

ii. AIUM and ACR Guidelines for Performance of the MSK US Examination

2. Independent scanning

Unit 5 SPORTS US Examination of the Shoulder

1. Shoulder US Scanning Protocol

A. Instruction and supervised practice

B. Resources

i. SPORTS US Scanning Protocol ChecklistsShoulder

ii. AIUM Guidelines for Performance of the Shoulder Ultrasound Examination

iii. AIUM and ACR Guidelines for Performance of the MSK US Examination

2. Independent scanning

Unit 6 US Guided Interventional Procedures

1. Didactic instruction and discussion

A. Rationale for US-guided procedures

B. Principles of US-guided procedures

i. Patient selection

ii. Ergonomics

iii. Aseptic technique

iv. In-plane and out-of-plane needle tracking

v. Image optimisation for needle location, relocation and dynamic tracking, including transducer manipulation: translation (sliding), rotation, heel toe, tilting (toggling) and compression

vi. Recognising and correcting 'cross-cut' artefact when needle tracking

C. Specific applications and techniques

i. Joint, tendon sheath, nerve, ligament, bursa/cyst

ii. Use of 'stand-off'/oblique stand-off, hydrodissection, lavage and aspiration

2. Demonstration, discussion and practice using unembalmed cadaveric specimens, phantoms, turkey breasts, pig feet, pigs legs, firm tofu or other appropriate medium

A. In-plane and out-of-plane needle location and tracking

B. Needle relocation

C. Cross-cut artefact

D. Commonly performed US-guided procedures. It is strongly recommended that these procedures be practiced on an unembalmed cadaveric specimen. However, if this is not feasible, then fellows should practice all aspects of needle visualisation and tracking using other appropriate medium, and the principles of the procedures listed below reviewed in a formal didactic setting.

i. Shoulder

1. Subacromial-subdeltoid bursa injection

2. Intra-articular glenohumeral joint injection

3. Intra-articular acromioclavicular joint injection

4. Bicipital tendon sheath/groove injection

ii. Elbow

1. Intra-articular elbow joint injection 
2. Peritendinous or intratendinous injection of the common extensor tendon origin

3. Peritendinous or intratendinous injection of the common flexor tendon origin

iii. Wrist-Hand

1. Carpal tunnel injection

2. First dorsal compartment tendon sheath injection

3. Intra-articular wrist injection

iv. Hip

1. Intra-articular hip injection

2. Greater trochanteric bursa injection

3. Gluteus medius or minimus peritendinous or intratendinous injection

v. Knee

1. Intra-articular knee injection

2. Iliotibial band/bursa (distal) injection

vi. Ankle-Foot

1. Intra-articular tibiotalar joint injection

2. Peroneal tendon sheath injection

3. Periplantar or intraplantar fascia injection

vii. Miscellaneous

1. Aspiration or injection of a cyst

\section{Didactic practice sessions}

Didactic practice sessions should be scheduled with a qualified mentor on regular basis throughout the fellowship. A qualified mentor is one who has met the requirements outlined in the AIUM Training Guidelines for Physicians and Chiropractors Who Evaluate and Interpret Diagnostic Musculoskeletal Ultrasound Examinations (http://www.aium.org). During these sessions the fellow should apply the knowledge and skills acquired during the didactic instructional sessions in a controlled and supervised educational environment. The didactic practice sessions should include the following:

1. Practice and demonstration of performing a complete ultrasound evaluation of each major region listed in the scanning protocols including proper image optimisation and acquisition (see online supplementary appendix 1).

2. Practice and demonstration of proper image labelling and storage. Transference of images should follow the guidelines outlined by the Health Insurance Portability and Accountability Act (HIPAA).

3. Review of saved images from the fellow's self-directed practice scanning sessions and provision of constructive feedback regarding study completeness, and proper image optimisation, labeling, storage and transfer. Deficiencies should be reconciled during subsequent scanning sessions.

4. Practice and demonstration of interventional skills, preferably using unembalmed cadaveric specimens. If cadaveric specimens are not available, the fellow should practice appropriate imaging of target structures on live models, and should practice needle imaging and guidance techniques using turkey breasts, pig feet, pig legs, firm tofu, phantoms or other appropriate medium. As the fellow's skills improve, more advanced SPORTS US examination techniques and interventional procedures should be introduced into the didactic practice sessions (eg, hydrodissection, percutaneous treatment of calcific tendinosis, etc).

\section{Mentored clinical experience}

The fellow should have regularly scheduled clinical time in which they receive supervised hands-on experience performing diagnostic and interventional SPORTS US on patients. During this experience, fellows should practice and eventually demonstrate competency in all aspects of SPORTS US outlined in the learning objectives. Special attention should be paid to obtaining proficiency in performing the core competency diagnostic ultrasound examinations of the pathological conditions and ultrasound-guided procedures listed in the learning objectives. Determining competence will be discussed further in Record keeping and competency.

This component of the fellow's SPORTS US training process is required to ensure that the fellow can proficiently perform the core diagnostic and interventional SPORTS US competencies in clinical practice.

\section{Supplementary and continuing SPORTS US education}

The fellow's SPORTS US education should not be restricted to the formal educational activities outlined in Didactic instructional sessions through Mentored clinical experience. The fellow should be required to participate in independent practice scanning, during which time they can practice diagnostic scanning techniques, positioning for procedures, and scanning protocols using volunteers. During this time, the fellow should also acquire studies for review with their mentor, as previously discussed. The fellow should also be required to independently practice ultrasound-guided needle tracking using the appropriate medium (eg, cadaver, phantom, etc).

In addition to the above required supplementary and continuing SPORTS US education experiences, as time and resources allow, the fellow should be encouraged to participate in one or more of the following:

1. Reading SPORTS US journals and texts on a regular basis

2. Reviewing SPORTS US-related articles on regular basis. It is recommended that the fellow present a SPORTS US-related journal article during journal club at least on a quarterly basis

3. Participating in online SPORTS US related courses or DVD's

4. Reading online SPORTS US related educational material

5. Attending SPORTS US related conferences

\section{Record keeping and competency}

The fellow should maintain detailed records of all SPORTS US educational activities in which they participate throughout the fellowship. The fellow should also maintain a procedure log of all diagnostic and interventional SPORTS US procedures, including their role in the procedure (eg, observation, performance, interpretation or reporting). Detailed recording keeping serves multiple purposes

1. Assists with credentialing

2. Assists in practice accreditation

3. Supports application for certification examinations

Although maintaining records of the type and number of diagnostic and interventional ultrasound procedures is important, performing a specific number of ultrasound procedures does not necessarily determine competence. A milestone system is a more appropriate way of determining competence and is in agreement with graduate medical education competency assessment recommendations by the Accreditation Council for Graduate Medical Education. Milestones use a five-point ordinal scale of escalating skill level, with competence determined when a level three or higher has been achieved. Milestones for each learning objective in the SPORTS US should be developed, and the fellow should achieve competence in all of the milestones on completion of their fellowship. Sample diagnostic and interventional ultrasound milestones are provided in online supplementary appendix 2 . 
Finally, it is recommended that an objective written and practical test be developed to assist with assessing the sports medicine fellow's SPORTS US knowledge and skill.

\section{What are the new findings?}

This document provides sports medicine fellowship directors and those interested in sports ultrasound education with a guideline for creating a SPORTS US curriculum.

\section{How might it impact on clinical practice in the near} future?

Completing this curriculum will prepare sports medicine physicians to proficiently practice the core competencies of SPORTS US.

\section{Author affiliations}

${ }^{1}$ Department of Physical Medicine and Rehabilitation, University of California Davis School of Medicine, Sacramento, California, USA

${ }^{2}$ Department of Physical Medicine and Rehabilitation, Mayo Clinic Sports Medicine Center, Mayo Clinic School of Medicine, Rochester, Minnesota, USA

${ }^{3}$ Department of Orthopedics, University of North Carolina, Chapel Hill, North Carolina, USA

${ }^{4}$ University of New Hampshire, Durham, New Hampshire, USA

Seacoast Orthopedics and Sports Medicine, Somersworth, New Hampshire, USA

${ }^{5}$ Division of Sports Medicine and Non-Operative Orthopaedics, Department of Family
Medicine and Orthopaedics, David Geffen School of Medicine, University of California, Los Angeles, California, USA

${ }^{6}$ Department of Orthopaedics and Rehabilitation, Department of Family Medicine, University of lowa Sports Medicine, lowa City, lowa, USA

${ }^{7}$ Departments of Family Medicine and Orthopaedics and Sports Medicine, University of Washington, Seattle, Washington, USA

${ }^{8}$ York Hospital Sports Medicine Fellowship Program, York, Pennsylvania, USA

${ }^{9}$ Department of Family and Community Medicine, Pennsylvania State University College of Medicine, Hershey, Pennsylvania, USA

${ }^{10}$ Department of Family, Community, and Preventive Medicine, Drexel University College of Medicine, Philadelphia, Pennsylvania, USA

${ }^{11} \mathrm{HQ}$ Air Armament Center, Eglin Air Force Base Family Medicine Residency, Florida, USA

${ }^{12}$ Department of Physical Medicine and Rehabilitation, Mayo Clinic College of

Medicine, Mayo Clinic Sports Medicine Center, Rochester, Minnesota, USA

${ }^{13}$ Department of Radiology, Mayo Clinic College of Medicine, Mayo Clinic, Rochester, Minnesota, USA

${ }^{14}$ University of Kansas School of Medicine-Wichita, Wichita, Kansas, USA

Contributors All authors were involved in the initial planning of the curriculum. The structure and content of the entire curriculum was discussed. The initial draft of the document was then created by JTF. The document was subsequently distributed to all of the other authors (DB, FB, JD, MMH, KH, ML, SM, JS, MS) for review. After all of the authors reviewed the document, the authors again collectively discussed further revisions. The suggested revisions were then performed by JTF. The document was reviewed by all of the other authors (DB, FB, JD, MMH, KH, ML, SM, JS, MS) one final time before submission.

Competing interests None.

Provenance and peer review Not commissioned; externally peer reviewed.

\section{REFERENCE}

1 Finnoff J, Lavallee ME, Smith J. Musculoskeletal ultrasound education for sports medicine fellows: a suggested/potential curriculum by the American Medical Society for Sports Medicine. Br J Sports Med 2010;44:1144-8. 